\title{
A CRITICAL FRACTIONAL LAPLACE EQUATION IN THE RESONANT CASE
}

\author{
RAFFAELla SERVADEI
}

ABStRaCt. In this paper we complete the study of the following non-local fractional equation involving critical nonlinearities

$$
\begin{cases}(-\Delta)^{s} u-\lambda u=|u|^{2^{*}-2} u & \text { in } \Omega, \\ u=0 & \text { in } \mathbb{R}^{n} \backslash \Omega,\end{cases}
$$

started in the recent papers [13], [17]-[19]. Here $s \in(0,1)$ is a fixed parameter, $(-\Delta)^{s}$ is the fractional Laplace operator, $\lambda$ is a positive constant, $2^{*}=2 n /(n-2 s)$ is the fractional critical Sobolev exponent and $\Omega$ is an open bounded subset of $\mathbb{R}^{n}, n>2 s$, with Lipschitz boundary. Aim of this paper is to study this critical problem in the special case when $n \neq 4 s$ and $\lambda$ is an eigenvalue of the operator $(-\Delta)^{s}$ with homogeneous Dirichlet boundary datum. In this setting we prove that this problem admits a non-trivial solution, so that with the results obtained in [13], [17]-[19], we are able to show that this critical problem admits a nontrivial solution provided

- $n>4 s$ and $\lambda>0$,

- $n=4 s$ and $\lambda>0$ is different from the eigenvalues of $(-\Delta)^{s}$,

- $2 s<n<4 s$ and $\lambda>0$ is sufficiently large.

In this way we extend completely the famous result of Brezis and Nirenberg (see [4], [5], [9], [23]) for the critical Laplace equation to the non-local setting of the fractional Laplace equation.

2010 Mathematics Subject Classification. Primary: 49J35, 35A15, 35S15; Secondary: 47G20, 45G05.

Key words and phrases. Critical nonlinearities, best critical Sobolev constant, variationaltechniques, Linking Theorem, integrodifferentialoperators, fractional Laplacian.

Supported by the MIUR National Research Project Variational and Topological Methods in the Study of Nonlinear Phenomenaand by the ERC grant $\varepsilon$ (Elliptic PDE's and Symmetry of Interfaces and Layers for Odd Nonlinearities). 


\section{Introduction}

The Yamabe problem for the Laplace operator (or, more generally, for uniformly elliptic operators) was widely studied in the literature (see, [10], [20], [22] and references therein). Recently, also in the non-local framework many papers concerning critical equationshas appeared, both for pure mathematical interest and for the various applications in many fields (such as, e.g. optimization, finance, phase transitions, stratified materials, anomalous diffusion, crystal dislocation, soft thin films, semipermeable membranes, flame propagation, conservation laws, ultra-relativisticlimits of quantum mechanics, quasi-geostrophic flows, multiple scattering, minimal surfaces, materials science and water waves). In the nonlocal framework, for instance, in [3], [21] the authors study a critical problem driven by a non-local operator defined as the power of the Laplacian, while in [13], [17]-[19] critical problems driven by the fractional Laplace operator $(-\Delta)^{s}$ are considered.

Aim of this paper is to complete the study carried on in [13], [17]-[19], where the existence of a nontrivial solution for the problem:

$$
\begin{cases}(-\Delta)^{s} u-\lambda u=|u|^{2^{*}-2} u & \text { in } \Omega, \\ u=0 & \text { in } \mathbb{R}^{n} \backslash \Omega,\end{cases}
$$

was established in the special case when the parameter $\lambda>0$ is different from the eigenvalues of $(-\Delta)^{s}$. Here $s \in(0,1)$ is fixed and $-(-\Delta)^{s}$ is the fractional Laplace operator, which (up to normalization factors) may be defined as

$$
-(-\Delta)^{s} u(x)=\int_{\mathbb{R}^{n}} \frac{u(x+y)+u(x-y)-2 u(x)}{|y|^{n+2 s}} d y, \quad x \in \mathbb{R}^{n},
$$

the set $\Omega \subset \mathbb{R}^{n}, n>2 s$, is open, bounded and with Lipschitz boundary, $\lambda>0$ and $2^{*}=2 n /(n-2 s)$ is the fractional critical Sobolev exponent (notice that when $s=1$ the above exponent reduces to the classical critical Sobolev exponent).

The homogeneous Dirichlet datum in (1.1) is given in $\mathbb{R}^{n} \backslash \Omega$ and not simply on $\partial \Omega$, as it happens in the classical case of the Laplacian, consistently with the non-local nature of the operator $(-\Delta)^{s}$. In the recent works [13], [17]-[19] we proved that the famous result by Brezis and Nirenberg (see [4], [5], [9], [23]) for the Laplace equation continues to hold also in the nonlocal setting of (1.1), provided $\lambda$ is not an eigenvalue of $(-\Delta)^{s}$.

With respect to the classical Brezis-Nirenberg result in these papers it remains open the resonant case in dimension different from $4 s$, that is the case when $n \neq 4 s$ and $\lambda$ is an eigenvalue of the operator $(-\Delta)^{s}$ with homogeneous Dirichlet boundary data. Aim of this paper is to consider (1.1) in this setting, since we though that it is interesting to check what happens in this case in order 
to verify if the classical result known for the Laplacian can be extended to the non-local fractional framework. In this way the study of the critical fractional Laplace problem is completed. The main result we obtain in the present paper is the following:

THEOREM 1.1. Let $s \in(0,1), n>2 s$ and $\Omega$ be an open bounded set of $\mathbb{R}^{n}$ with Lipschitz boundary. Moreover, let $\lambda$ be an eigenvalue of $(-\Delta)^{s}$ with homogeneous Dirichlet boundary data. Then, problem (1.1) admits a weaksolution $u \in H^{s}\left(\mathbb{R}^{n}\right)$, which is not identically zero, and such that $u=0$ almost everywhere in $\mathbb{R}^{n} \backslash \Omega$, provided that either

(a) $n>4 s$, or

(b) $2 s<n<4 s$ and $\lambda$ is sufficiently large.

As a consequence of Theorem 1.1 and of [13, Theorem 1.2], [17, Theorem 4] and [18, Theorem 1] we get the following existence result, which extends completely to the non-local fractional framework the well-known Brezis-Nirenberg type results given in [4], [5], [9], [23] for the Laplace equation:

TheOREM 1.2. Let $s \in(0,1), n>2 s$ and $\Omega$ be an open bounded set of $\mathbb{R}^{n}$ with Lipschitz boundary. Then, problem (1.1) admits a weaksolution $u \in H^{s}\left(\mathbb{R}^{n}\right)$, which is not identically zero, and such that $u=0$ almost everywhere in $\mathbb{R}^{n} \backslash \Omega$, provided that either

(a) $n>4 s$ and $\lambda>0$, or

(b) $n=4 s$ and $\lambda>0$ is different from the eigenvalues of $(-\Delta)^{s}$, or

(c) $n<4$ s and $\lambda>0$ is sufficiently large.

Roughly speaking, Theorem 1.2 says that what happens in the non-local framework is exactly what we know in the classing setting (see [4], [5], [9], [23] and also [10], [20], [22] and references therein; of course the extension from the local setting to the non-local one is not straightforward as we will see in the course of the proofs).

We would like to note that, as it happens in the Laplacian case when $n=4$, also in the non-local framework there is a dimension $(n=4 s)$ where resonance creates problem. Also, when $s=1$ (which corresponds to the Laplace case) these two dimensions are the same.

In the classical setting of the Laplacian this fact was not underlined in the original paper of Capozzi, Fortunato and Palmieri (see [5]), but it was noticed by Zhang in [23]. For an explanation of this strange phenomenon see also [2] and $[8]$.

In order to prove Theorem 1.1 we mainly use the fact that problem (1.1) is variational in nature and its weak formulation is given by 


$$
\left\{\begin{array}{r}
\int_{\mathbb{R}^{2 n}} \frac{(u(x)-u(y))(\varphi(x)-\varphi(y))}{|x-y|^{n+2 s}} d x d y-\lambda \int_{\Omega} u(x) \varphi(x) d x \\
=\int_{\Omega}|u(x)|^{2^{*}-2} u(x) \varphi(x) d x \\
\text { for all } \varphi \in H^{s}\left(\mathbb{R}^{n}\right) \text { with } \varphi=0 \text { a.e. in } \mathbb{R}^{n} \backslash \Omega, \\
u \in H^{s}\left(\mathbb{R}^{n}\right) \text { with } u=0 \text { a.e. in } \mathbb{R}^{n} \backslash \Omega .
\end{array}\right.
$$

Problem (1.3) represents the Euler-Lagrange of the functional $\mathcal{J}_{s, \lambda}: X_{0} \rightarrow \mathbb{R}$ defined as

$$
\mathcal{J}_{s, \lambda}(u)=\frac{1}{2} \int_{\mathbb{R}^{2 n}} \frac{|u(x)-u(y)|^{2}}{|x-y|^{n+2 s}} d x d y-\frac{\lambda}{2} \int_{\Omega}|u(x)|^{2} d x-\frac{1}{2^{*}} \int_{\Omega}|u(x)|^{2^{*}} d x,
$$

where the functional space $X_{0}$ is the Hilbert space defined as

$$
X_{0}=\left\{u \in H^{s}\left(\mathbb{R}^{n}\right): u=0 \text { a.e. in } \mathbb{R}^{n} \backslash \Omega\right\}
$$

and endowed with the norm given by

$$
X_{0} \ni v \mapsto\|v\|_{X_{0}}=\left(\int_{\mathbb{R}^{2 n}} \frac{|v(x)-v(y)|^{2}}{|x-y|^{n+2 s}} d x d y\right)^{1 / 2} .
$$

For this see [15, Lemma 7], while for a general definition of $X_{0}$ and its propertieswe refer to [13]-[17] and [19]. Here $H^{s}\left(\mathbb{R}^{n}\right)$ is the usual fractional Sobolev space (for this see, for instance, [7]), endowed with the so-called Gagliardo norm

$$
\|g\|_{H^{s}\left(\mathbb{R}^{n}\right)}=\|g\|_{L^{2}\left(\mathbb{R}^{n}\right)}+\left(\int_{\mathbb{R}^{2 n}} \frac{|g(x)-g(y)|^{2}}{|x-y|^{n+2 s}} d x d y\right)^{1 / 2} .
$$

Thus, in looking for weak solutions of (1.1) (that is solutions of problem (1.3)) we study the critical points of the functional $\mathcal{J}_{s, \lambda}$ using classical minimax theorems, namely the Linking Theorem (see, e.g. [1], [11], [12]). In order to apply such critical points theorem to $\mathcal{J}_{s, \lambda}$ we argue as in [13], [17], [18]. In particular, since the functional $\mathcal{J}_{s, \lambda}$ is not compact (due to the fact that $H^{s}\left(\mathbb{R}^{n}\right)$ is not compactly embedded into the critical Lebesgue space $L^{2^{*}}\left(\mathbb{R}^{n}\right)$ ), the Palais-Smale condition does not hold globally, but only in a suitable range related to the best constant in the embedding $H^{s}\left(\mathbb{R}^{n}\right) \hookrightarrow L^{2^{*}}\left(\mathbb{R}^{n}\right)$. For this reason, in order to apply the minimax theorem, we need to estimate the critical level of $\mathcal{J}_{s, \lambda}$ and, for proving such a estimate, we need to construct an explicit solution of the following limiting problem

$$
(-\Delta)^{s} u=|u|^{2^{*}-2} u \text { in } \mathbb{R}^{n} .
$$

For more details on this see Subsection 2.1. The paper is organized as follows. In Section 2 we will deal with the variational formulation of the problem. After introducing some notations and recalling some preliminary results, we will discuss the geometric and compactness properties of the functional $\mathcal{J}_{s, \lambda}$. Section 3 
will be devoted to the estimate of the minimax critical level of $\mathcal{J}_{s, \lambda}$ and to the proof of Theorem 1.1.

\section{The variational formulation of the problem}

As we said in the Introduction, problem (1.1) has a variational structure. Hence, in order to look for weak solutions of problem (1.1) we study the critical points of the functional $\mathcal{J}_{s, \lambda}$ using classical minimax theorems. First of all, note that this functional is well defined thanks to [15, Lemma 8] and [17, Lemma 9]. Moreover, $\mathcal{J}_{s, \lambda}$ is Fréchet differentiable in $u \in X_{0}$ and for any $\varphi \in X_{0}$

$$
\begin{aligned}
\left\langle\mathcal{J}_{s, \lambda}^{\prime}(u), \varphi\right\rangle=\int_{\mathbb{R}^{2 n}} \frac{(u(x)-u(y))(\varphi(x)-\varphi(y))}{|x-y|^{n+2 s}} d x d y & \\
& -\lambda \int_{\Omega} u(x) \varphi(x) d x-\int_{\Omega}|u(x)|^{2^{*}-2} u(x) \varphi(x) d x .
\end{aligned}
$$

Before proving Theorem 1.1 we need some notation and some preliminary results.

2.1. Notations and preliminary results. In the sequel we will denote by $\left(\lambda_{k, s}\right)_{k \in \mathbb{N}}$ the sequence of eigenvalues of the operator $(-\Delta)^{s}$, with homogeneous Dirichlet boundary data, such that

$$
0<\lambda_{1, s}<\lambda_{2, s} \leq \ldots \leq \lambda_{k, s} \leq \lambda_{k+1, s} \leq \ldots \quad \text { and } \quad \lambda_{k, s} \rightarrow+\infty \quad \text { as } k \rightarrow+\infty .
$$

Moreover, $e_{k, s}$ will be the eigenfunction corresponding to $\lambda_{k, s}$ for any $k \in \mathbb{N}$ and

$$
\mathbb{P}_{k+1, s}:=\left\{u \in X_{0} \text { such that }\left\langle u, e_{j, s}\right\rangle_{X_{0}}=0 \text { for all } j=1, \ldots, k\right\},
$$

where $\langle\cdot, \cdot\rangle_{X_{0}}$ is the scalar product on $X_{0}$ defined as

$$
\langle u, v\rangle_{X_{0}}=\int_{\mathbb{R}^{2 n}} \frac{(u(x)-u(y))(v(x)-v(y))}{|x-y|^{n+2 s}} d x d y .
$$

For a complete study of the eigenvalues and the eigenfunctions of $(-\Delta)^{s}$ (and, more generally, of non-local integrodifferential operators) we refer to [16, Proposition 9 and Appendix A]. Here we also need to introduce the best fractional critical Sobolev constant $S_{s}$ for the embedding of $H^{s}\left(\mathbb{R}^{n}\right)$ into $L^{2^{*}}\left(\mathbb{R}^{n}\right)$ defined as

$$
\begin{aligned}
& S_{s}:=\inf _{v \in H^{s}\left(\mathbb{R}^{n}\right) \backslash\{0\}} S_{s}(v), \\
& H^{s}\left(\mathbb{R}^{n}\right) \backslash\{0\} \ni v \mapsto S_{s}(v):=\frac{\int_{\mathbb{R}^{2 n}} \frac{|v(x)-v(y)|^{2}}{|x-y|^{n+2 s}} d x d y}{\left(\int_{\mathbb{R}^{n}}|v(x)|^{2^{*}} d x\right)^{2 / 2^{*}}} .
\end{aligned}
$$

With this, we can recall the following result obtained in [6, Theorem 1.1]: 
Proposition 2.1. The infimum in formula (2.2) is attained, that is

$$
S_{s}=S_{s}(\widetilde{u})
$$

where

$$
\widetilde{u}(x)=\kappa\left(\mu^{2}+\left|x-x_{0}\right|^{2}\right)^{-(n-2 s) / 2}, \quad x \in \mathbb{R}^{n}
$$

with $\kappa \in \mathbb{R} \backslash\{0\}, \mu>0$ and $x_{0} \in \mathbb{R}^{n}$ fixed constants. Equivalently, the function $\bar{u}$ defined as

$$
\bar{u}(x)=\frac{\widetilde{u}(x)}{\|\widetilde{u}\|_{L^{2 *}\left(\mathbb{R}^{n}\right)}}
$$

is such that

$$
S_{s}=\inf _{\substack{v \in H^{s}\left(\mathbb{R}^{n}\right) \\\|v\|_{L^{2 *}\left(\mathbb{R}^{n}\right)}=1}} \int_{\mathbb{R}^{2 n}} \frac{|v(x)-v(y)|^{2}}{|x-y|^{n+2 s}} d x d y=\int_{\mathbb{R}^{2 n}} \frac{|\bar{u}(x)-\bar{u}(y)|^{2}}{|x-y|^{n+2 s}} d x d y
$$

In the sequel we suppose that, up to a translation, $x_{0}=0$ in (2.5). Arguing as in $[17$, Section 4$]$, we consider the function

$$
u^{*}(x)=\bar{u}\left(\frac{x}{S_{s}^{1 /(2 s)}}\right), \quad x \in \mathbb{R}^{n},
$$

which is an explicit solution of the limiting problem

$$
(-\Delta)^{s} u=|u|^{2^{*}-2} u \quad \text { in } \mathbb{R}^{n}
$$

such that $\left\|u^{*}\right\|_{L^{2^{*}\left(\mathbb{R}^{n}\right)}}^{2^{*}}=S_{s}^{n /(2 s)}$ and also the functions

$$
\begin{array}{ll}
U_{\varepsilon}(x)=\varepsilon^{-(n-2 s) / 2} u^{*}(x / \varepsilon), & x \in \mathbb{R}^{n}, \\
u_{\varepsilon}(x)=\eta(x) U_{\varepsilon}(x), & x \in \mathbb{R}^{n},
\end{array}
$$

for any $\varepsilon>0$. Here $\eta \in C^{\infty}\left(\mathbb{R}^{n}\right)$ is a function such that $0 \leq \eta \leq 1 \mathrm{in} \mathbb{R}^{n}, \eta \equiv 1$ in $B_{\delta}$ and $\eta \equiv 0$ in $\mathcal{C} B_{2 \delta}$, where $B_{\delta}=B(0, \delta)$ and $\mathcal{C} B_{\delta}=\mathbb{R}^{n} \backslash B_{\delta}$, with $\delta>0$ fixed so that $B_{4 \delta} \subset \Omega$.

Note that $u_{\varepsilon} \in X_{0}$ and $u_{\varepsilon}=0$ almost everywhere in $\mathbb{R}^{n} \backslash \Omega$. What is important about $u_{\varepsilon}$ is that this function satisfies some crucial estimates that we recall here below (for a proof see [17, Propositions 21 and 22 and Subsection 4.2.1] and [13, Proposition 7.2]):

Proposition 2.2. Let $s \in(0,1)$ and $n>2 s$. Then the following estimates hold true:

$$
\int_{\mathbb{R}^{2 n}} \frac{\left|u_{\varepsilon}(x)-u_{\varepsilon}(y)\right|^{2}}{|x-y|^{n+2 s}} d x d y \leq S_{s}^{n /(2 s)}+\mathcal{O}\left(\varepsilon^{n-2 s}\right)
$$




$$
\begin{aligned}
\int_{\mathbb{R}^{n}}\left|u_{\varepsilon}(x)\right|^{2} d x & \geq \begin{cases}C_{s} \varepsilon^{2 s}+\mathcal{O}\left(\varepsilon^{n-2 s}\right) & \text { if } n>4 s, \\
C_{s} \varepsilon^{2 s}|\log \varepsilon|+\mathcal{O}\left(\varepsilon^{2 s}\right) & \text { if } n=4 s, \\
C_{s} \varepsilon^{n-2 s}+\mathcal{O}\left(\varepsilon^{2 s}\right) & \text { if } n<4 s,\end{cases} \\
\int_{\mathbb{R}^{n}}\left|u_{\varepsilon}(x)\right|^{2^{*}} d x & =S_{s}^{n /(2 s)}+\mathcal{O}\left(\varepsilon^{n}\right), \\
\int_{\mathbb{R}^{n}}\left|u_{\varepsilon}(x)\right|^{2^{*}-1} d x & =\mathcal{O}\left(\varepsilon^{(n-2 s) / 2}\right),
\end{aligned}
$$

and

$$
\int_{\mathbb{R}^{n}}\left|u_{\varepsilon}(x)\right| d x=\mathcal{O}\left(\varepsilon^{(n-2 s) / 2}\right),
$$

as $\varepsilon \rightarrow 0$, for some positive constant $C_{s}$ depending on $s$.

2.2. Geometric and compactness condition of the functional $\mathcal{J}_{s, \lambda}$. In the present paper we are interested in problem (1.1), when the parameter $\lambda$ is an eigenvalue of $(-\Delta)^{s}$, say when $\lambda=\lambda_{k, s}$ for some $k \in \mathbb{N}$. Without loss of generality, we can assume that $\lambda_{k, s}$ has multiplicity $h \in \mathbb{N}$ and that

$$
\lambda_{k-1, s}<\lambda_{k, s}=\lambda_{k+1, s}=\ldots=\lambda_{k+h-1, s}<\lambda_{k+h, s} .
$$

In order to prove Theorem 1.1 our strategy will consist in applying the Linking Theorem (see [11], [12]) to the functional $\mathcal{J}_{s, \lambda_{k, s}}$, that is $\mathcal{J}_{s, \lambda}$ with $\lambda=\lambda_{k, s}$. As it is well-known, the main ingredients of this minimax theorem are a suitable geometric structure and a compactness condition, namely the Palais-Smale condition at level $c \in \mathbb{R}$, given by:

every Palais-Smale sequence $\left(u_{j}\right)_{j \in \mathbb{N}}$ in $X_{0}$ at level $c \in \mathbb{R}$ admits a subsequence strongly convergent in $X_{0}$.

We say that $\left(u_{j}\right)_{j \in \mathbb{N}}$ in $X_{0}$ is a Palais-Smale sequence for $\mathcal{J}_{s, \lambda}$ at level $c \in \mathbb{R}$ if

$$
\mathcal{J}_{s, \lambda}\left(u_{j}\right) \rightarrow c \text { and } \sup \left\{\left|\left\langle\mathcal{J}_{s, \lambda}^{\prime}\left(u_{j}\right), \varphi\right\rangle\right|: \varphi \in X_{0},\|\varphi\|_{X_{0}}=1\right\} \rightarrow 0 \text { as } j \rightarrow+\infty .
$$

By $\left[18\right.$, Proposition 10] we know that the functional $\mathcal{J}_{s, \lambda}$ satisfies the geometric features required by the Linking Theorem, when $\lambda \geq \lambda_{1, s}$. Hence, in particular, $\mathcal{J}_{s, \lambda_{k, s}}$ has the geometric structure of the Linking Theorem, that is the following proposition is valid:

Proposition 2.3. There exist $\rho>0$ and $\beta>0$ such that

(a) for any $u \in \mathbb{P}_{k+1, s}$ with $\|u\|_{X_{0}}=\rho$ it results that $\mathcal{J}_{s, \lambda_{k, s}}(u) \geq \beta$;

(b) $\mathcal{J}_{s, \lambda_{k, s}}(u) \leq 0$ for any $u \in \operatorname{span}\left\{e_{1, s}, \ldots, e_{k, s}\right\}$;

(c) for any finite dimensional subspace $\mathbb{F}$ of $X_{0}$, there exists $R>\rho$ such that

$$
\sup _{\substack{u \in \mathbb{F} \\\|u\|_{X_{0}} \geq R}} \mathcal{J}_{s, \lambda_{k, s}}(u) \leq 0
$$


In particular, we can construct $\mathbb{F}$ as follows:

$$
\mathbb{F}=\mathbb{F}_{\varepsilon}:=\operatorname{span}\left\{e_{1, s}, \ldots, e_{k, s}\right\} \oplus \operatorname{span}\left\{\widetilde{z}_{\varepsilon}\right\},
$$

with $\widetilde{z}_{\varepsilon}=z_{\varepsilon} /\left\|z_{\varepsilon}\right\|_{X_{0}}, z_{\varepsilon}=u_{\varepsilon}-\sum_{i=1}^{k}\left(\int_{\Omega} u_{\varepsilon}(x) e_{i, s}(x) d x\right) e_{i, s}$ and $u_{\varepsilon}$ as in (2.9) for $\varepsilon>0$.

Moreover, by [17, Proposition 5] (which holds true for any $\lambda>0$ ) and the fact that $\lambda_{k, s} \geq \lambda_{1, s}>0$ (see [16, Proposition 9a)]) the functional $\mathcal{J}_{s, \lambda_{k, s}}$ also verifies the Palais-Smale condition up to a suitable threshold, i.e. the following result holds true:

Proposition 2.4. Let $c \in \mathbb{R}$ be such that

$$
c<\frac{s}{n} S_{s}^{n /(2 s)},
$$

where $S_{s}$ is the constant defined in (2.2). Then, the functional $\mathcal{J}_{s, \lambda_{k, s}}$ satisfies the Palais-Smale condition at level $c$.

Roughly speaking, Proposition 2.4 says that the functional $\mathcal{J}_{s, \lambda_{k, s}}$ satisfies the Palais-Smale condition only below a suitable threshold related to the fractional critical Sobolev constant. Since this condition does not hold globally, in order to apply the Linking Theorem to $\mathcal{J}_{s, \lambda_{k, s}}$, we need to estimate the critical level of this functional and show that it stays below the threshold where the Palais-Smale condition is satisfied. This will be done in the next section.

\section{Estimate of the minimax critical level}

This section is devoted to the estimate of the minimax critical level of the functional $\mathcal{J}_{s, \lambda_{k, s}}$. Here, in some sense, we argue as in [13, Subsection 7.2] and in [18, Section 7] even if, with respect to these papers, some differences arise, due to the fact that here we consider the resonant case, that is the case when $\lambda$ is an eigenvalue of $(-\Delta)^{s}$. In fact, here the estimates are more delicate: on this we will be more precise in the sequel. The Linking critical level of $\mathcal{J}_{s, \lambda_{k, s}}$ is given by

$$
c_{\varepsilon, \lambda_{k, s}}=\inf _{h \in \Gamma} \max _{u \in Q} \mathcal{J}_{s, \lambda_{k, s}}(h(u)),
$$

where

$$
\begin{aligned}
\Gamma & =\left\{h \in C\left(\bar{Q} ; X_{0}\right): h=\mathrm{id} \text { on } \partial Q\right\}, \\
Q & =\left(\bar{B}_{R} \cap \operatorname{span}\left\{e_{1, s}, \ldots, e_{k, s}\right\}\right) \oplus\left\{r \widetilde{z}_{\varepsilon}: r \in(0, R)\right\},
\end{aligned}
$$

and $R$ and $\widetilde{z}_{\varepsilon}$ are as in Proposition 2.3. In order to estimate $c_{\varepsilon, \lambda_{k, s}}$, we will use the particular choice of $\mathbb{F}=\mathbb{F}_{\varepsilon}$ given in (2.11) (note that $Q \subset \mathbb{F}_{\varepsilon}$ ). We want to show the following result 
Proposition 3.1. Let $S_{s}$ be as in (2.2). Then,

$$
c_{\varepsilon, \lambda_{k, s}}<\frac{s}{n} S_{s}^{n /(2 s)}
$$

for $\varepsilon$ sufficiently small, provided $n>4 s$ or $n<4 s$ and $\lambda_{k, s}$ is large enough.

Proof. First of all, note thatby definition of $c_{\varepsilon, \lambda_{k, s}}$ for any $h \in \Gamma$

$$
c_{\varepsilon, \lambda_{k, s}} \leq \max _{u \in Q} \mathcal{J}_{s, \lambda_{k, s}}(h(u))
$$

and so, in particular, taking $h=$ id and using the fact that $Q \subset \mathbb{F}_{\varepsilon}$, we have

$$
c_{\varepsilon, \lambda_{k, s}} \leq \max _{u \in Q} \mathcal{J}_{s, \lambda_{k, s}}(u) \leq \max _{u \in \mathbb{F}_{\varepsilon}} \mathcal{J}_{s, \lambda_{k, s}}(u)
$$

Since $\mathbb{F}_{\varepsilon}$ is a linear space,

$$
\begin{aligned}
\max _{u \in \mathbb{F}_{\varepsilon}} \mathcal{J}_{s, \lambda_{k, s}}(u) & =\max _{\substack{u \in \mathbb{F}_{\varepsilon} \\
\zeta \neq 0}} \mathcal{J}_{s, \lambda_{k, s}}\left(|\zeta| \cdot \frac{u}{|\zeta|}\right) \\
& =\max _{\substack{u \in \mathbb{F}_{\varepsilon} \\
\zeta>0}} \mathcal{J}_{s, \lambda_{k, s}}(\zeta u) \leq \max _{\substack{u \in \mathbb{F}_{\varepsilon} \\
\zeta \geq 0}} \mathcal{J}_{s, \lambda_{k, s}}(\zeta u) .
\end{aligned}
$$

Hence, (3.2)-(3.4) yield that

$$
c_{\varepsilon, \lambda_{k}, s} \leq \max _{\substack{u \in \mathbb{F}_{\varepsilon} \\ \zeta \geq 0}} \mathcal{J}_{s, \lambda_{k, s}}(\zeta u)
$$

Then, in order to prove Proposition 3.1, by (3.5) it is enough to show that

$$
\max _{\substack{u \in \mathbb{F}_{\varepsilon} \\ \zeta \geq 0}} \mathcal{J}_{s, \lambda_{k}, s}(\zeta u)<\frac{s}{n} S_{s}^{n /(2 s)} .
$$

Note that, by [17, Proposition 20], for any $u \in X_{0} \backslash\{0\}$

$$
\max _{\zeta \geq 0} \mathcal{J}_{s, \lambda_{k, s}}(\zeta u)=\frac{s}{n}\left(\frac{\|u\|_{X_{0}}-\lambda_{k, s}\|u\|_{L^{2}(\Omega)}^{2}}{\|u\|_{L^{2^{*}}(\Omega)}^{2^{*}}}\right)^{n /(2 s)},
$$

and that the right-hand side in (3.7) is scale invariant. Hence, as a consequence of $(3.7)$, relation $(3.6)$ is equivalent to

$$
M_{\varepsilon}:=\max _{\substack{u \in \mathbb{F}_{\varepsilon} \\\|u\|_{L^{2}(\Omega)}=1}}\left(\|u\|_{X_{0}}^{2}-\lambda_{k, s}\|u\|_{L^{2}(\Omega)}^{2}\right)<S_{s},
$$

so that, in order to prove Proposition 3.1, it is enough to show that (3.8) holds true. Let us prove inequality (3.8). At this purpose, let us recall that, by [13, Proposition 7.3)] $M_{\varepsilon}$ is achieved in some $u_{M} \in \mathbb{F}_{\varepsilon}$, which can be written as follows $\left(^{1}\right)$

$$
u_{M}=\widetilde{v}+t z_{\varepsilon}
$$

$\left.{ }^{1}\right)$ Beware that $u_{M}, \widetilde{v}$ and $t$ (and also $v$ in Claim 2 below) depend on $\varepsilon$. For simplicity we omit this dependence in the notation. 
where $\widetilde{v} \in \operatorname{span}\left\{e_{1, s}, \ldots, e_{k, s}\right\}, t \geq 0$ and $z_{\varepsilon}$ is as in Proposition 2.3, and such that

$$
\left\|u_{M}\right\|_{L^{2^{*}}(\Omega)}=1 .
$$

For now on we proceed by steps.

Claim 1. There exists a positive constant $\bar{c}$ such that $t=t_{\varepsilon} \leq \bar{c}$ for $\varepsilon>0$ small enough.

Proof. First of all, note that, by the Hőlder inequality and the properties of $u_{M}$, we can bound $u_{M}$ as follows

$$
\left\|u_{M}\right\|_{L^{2}(\Omega)}^{2} \leq|\Omega|^{n /(2 s)}\left\|u_{M}\right\|_{L^{2^{*}}(\Omega)}^{2}=|\Omega|^{n /(2 s)} .
$$

Moreover, $\widetilde{v}$ and $z_{\varepsilon}$ are orthogonal in $L^{2}(\Omega)$ and in $X_{0}$, since the sequence $\left(e_{k, s}\right)_{k \in \mathbb{N}}$ of eigenfunctions corresponding to $\lambda_{k}$ is an orthonormal basis of $L^{2}(\Omega)$ and anorthogonal basis of $X_{0}$ (see [16, Proposition 9f)]). As a consequence of this, we get that

$$
\left\|u_{M}\right\|_{L^{2}(\Omega)}^{2}=\|\widetilde{v}\|_{L^{2}(\Omega)}^{2}+t_{\varepsilon}^{2}\left\|z_{\varepsilon}\right\|_{L^{2}(\Omega)}^{2} \geq\|\widetilde{v}\|_{L^{2}(\Omega)}^{2} .
$$

By (3.11) and (3.12), we easily get that both $\left\|u_{M}\right\|_{L^{2}(\Omega)}$ and $\|\widetilde{v}\|_{L^{2}(\Omega)}$ are bounded uniformly in $\epsilon$ by a suitable $\widetilde{c}>0$. Furthermore, by [18, Proposition 4] we know that $e_{i, s} \in L^{\infty}(\Omega)$ for any $i \in \mathbb{N}$, so that also $\widetilde{v} \in \operatorname{span}\left\{e_{1, s}, \ldots, e_{k, s}\right\}$ does. Hence, $\widetilde{v} \in L^{2^{*}}(\Omega)$, since $\Omega$ is bounded and, by the equivalence of the norms in a finite dimensional space,we also have

$$
\|\widetilde{v}\|_{L^{2^{*}(\Omega)}} \leq \widetilde{c} .
$$

Also, by Proposition 2.2 and again [18, Proposition 4] we have

$$
\left|\int_{\Omega} u_{\varepsilon}(x) e_{i, s}(x) d x\right| \leq\left\|u_{\varepsilon}\right\|_{L^{1}(\Omega)}\left\|e_{i, s}\right\|_{L^{\infty}(\Omega)}=\mathcal{O}\left(\varepsilon^{(n-2 s) / 2}\right)
$$

as $\varepsilon \rightarrow 0$. As a consequence, using the definition of $z_{\varepsilon}$ and again Proposition 2.2, we get

$$
\begin{aligned}
\left\|z_{\varepsilon}\right\|_{L^{2^{*}}(\Omega)} & \geq\left\|u_{\varepsilon}\right\|_{L^{2^{*}}(\Omega)}-\sum_{i=1}^{k}\left|\int_{\Omega} u_{\varepsilon}(x) e_{i, s}(x) d x\right|\left\|e_{i, s}\right\|_{L^{2^{*}}(\Omega)} \\
& =S_{s}^{(n-2 s) /(4 s)}+\mathcal{O}\left(\varepsilon^{(n-2 s) / 2}\right) \geq \frac{S_{s}^{(n-2 s) /(4 s)}}{2}
\end{aligned}
$$

for $\varepsilon$ sufficiently small. Then, by (3.9), the fact that $t=t_{\varepsilon} \geq 0$, (3.10) and (3.13), we have

$$
\frac{S_{s}^{(n-2 s) /(4 s)} t_{\varepsilon}}{2} \leq t_{\varepsilon}\left\|z_{\varepsilon}\right\|_{L^{2^{*}}(\Omega)} \leq\left\|u_{M}\right\|_{L^{2^{*}}(\Omega)}+\|\widetilde{v}\|_{L^{2^{*}}(\Omega)} \leq 1+\widetilde{c},
$$


for $\varepsilon$ small enough. Hence, $t_{\varepsilon}$ is bounded for $\varepsilon$ sufficiently small and this ends the proof of Claim 1.

Claim 2. The function $u_{M} \in \mathbb{F}_{\varepsilon}$ can be written as $u_{M}=v+P_{k} \widetilde{v}+t \widetilde{u}_{\varepsilon}$, where $t \geq 0$,

$$
\begin{gathered}
v=\sum_{i=1}^{k-1}\left(\int_{\Omega}\left(\widetilde{v}(x)-t u_{\varepsilon}(x)\right) e_{i, s}(x) d x\right) e_{i, s} \in \operatorname{span}\left\{e_{1, s}, \ldots, e_{k-1, s}\right\}, \\
\widetilde{u}_{\varepsilon}=u_{\varepsilon}-P_{k} u_{\varepsilon}
\end{gathered}
$$

and the map $X_{0} \ni w \mapsto P_{k} w$ denotes the projection of $w$ on the direction $e_{k, s}$, that is

$$
P_{k} w=\left(\int_{\Omega} w(x) e_{k, s}(x) d x\right) e_{k, s} .
$$

Moreover, there exists a positive constant $\bar{\kappa}$, independent of $\varepsilon$, such that

$$
\left|\int_{\Omega} \widetilde{u}_{\varepsilon}(x) v(x) d x\right|=\left|\int_{\Omega} u_{\varepsilon}(x) v(x) d x\right| \leq \bar{\kappa}\|v\|_{L^{2}(\Omega)}\left\|u_{\varepsilon}\right\|_{L^{1}(\Omega)},
$$

and

$$
\left|\int_{\mathbb{R}^{2 n}} \frac{\left(\widetilde{u}_{\varepsilon}(x)-\widetilde{u}_{\varepsilon}(y)\right)(v(x)-v(y))}{|x-y|^{n+2 s}} d x d y\right| \leq \bar{\kappa}\|v\|_{L^{2}(\Omega)}\left\|u_{\varepsilon}\right\|_{L^{1}(\Omega)}
$$

for any $\varepsilon>0$.

Proof. By (3.9) and the definition of $z_{\varepsilon}$ (as given in Proposition 2.3), it is easily seen that

$$
\begin{aligned}
u_{M} & =\sum_{i=1}^{k}\left(\int_{\Omega} \widetilde{v}(x) e_{i, s}(x) d x\right) e_{i, s}+t\left(u_{\varepsilon}-\sum_{i=1}^{k}\left(\int_{\Omega} u_{\varepsilon}(x) e_{i, s}(x) d x\right) e_{i, s}\right) \\
& =\sum_{i=1}^{k-1}\left(\int_{\Omega}\left(\widetilde{v}(x)-t u_{\varepsilon}(x)\right) e_{i, s}(x) d x\right) e_{i, s}+P_{k} \widetilde{v}+t\left(u_{\varepsilon}-P_{k} u_{\varepsilon}\right) \\
& =v+P_{k} \widetilde{v}+t \widetilde{u}_{\varepsilon},
\end{aligned}
$$

with $v$ and $\widetilde{u}_{\varepsilon}$ as in (3.14) and (3.15), respectively.Let us start showing that (3.16) holds true. For this, note that $v$ and $P_{k} u_{\varepsilon}$ are orthogonal in $L^{2}(\Omega)$, so that

$$
\int_{\Omega} \widetilde{u}_{\varepsilon}(x) v(x) d x=\int_{\Omega}\left(u_{\varepsilon}(x)-P_{k} u_{\varepsilon}\right) v(x) d x=\int_{\Omega} u_{\varepsilon}(x) v(x) d x,
$$

while the Hölder inequality and the equivalence of the norm in a finite dimensional space give

$$
\begin{aligned}
\left|\int_{\Omega} \widetilde{u}_{\varepsilon}(x) v(x) d x\right| & =\left|\int_{\Omega} u_{\varepsilon}(x) v(x) d x\right| \\
& \leq\left\|u_{\varepsilon}\right\|_{L^{1}(\Omega)}\|v\|_{L^{\infty}(\Omega)} \leq \bar{\kappa}\left\|u_{\varepsilon}\right\|_{L^{1}(\Omega)}\|v\|_{L^{2}(\Omega)}
\end{aligned}
$$


for a suitable $\bar{\kappa}>0$, independent of $\varepsilon$. Thus, (3.16) is proved. Now, let us show (3.17). At this purpose, we write

$$
v=\sum_{i=1}^{k-1} v_{i} e_{i, s}
$$

for some $v_{i} \in \mathbb{R}$, so that, again by [16, Proposition 9f)],

$$
\|v\|_{L^{2}(\Omega)}^{2}=\sum_{i=1}^{k-1} v_{i}^{2}
$$

By (3.18), the fact that $e_{i, s}$ is an eigenfunction of $(-\Delta)^{s}$ with eigenvalue $\lambda_{i, s}$ and the definition of scalar product in $X_{0}$ (see (2.1)), we have

$$
\begin{aligned}
\left\langle\widetilde{u}_{\varepsilon}, v\right\rangle_{X_{0}} & =\sum_{i=1}^{k-1} v_{i}\left\langle\widetilde{u}_{\varepsilon}, e_{i, s}\right\rangle_{X_{0}} \\
= & \sum_{i=1}^{k-1} \lambda_{i, s} v_{i} \int_{\Omega} \widetilde{u}_{\varepsilon}(x) e_{i, s}(x) d x=\sum_{i=1}^{k-1} \lambda_{i, s} v_{i} \int_{\Omega} u_{\varepsilon}(x) e_{i, s}(x) d x
\end{aligned}
$$

also thanks to the definition of $\widetilde{u}_{\varepsilon}$ and the orthogonality properties of $e_{i, s}$. So, by this and again the Hölder inequality, we get

$$
\left\langle\widetilde{u}_{\varepsilon}, v\right\rangle_{X_{0}}\left|\leq \sum_{i=1}^{k-1} \lambda_{i, s}\right| v_{i} \mid\left\|u_{\varepsilon}\right\|_{L^{1}(\Omega)}\left\|e_{i, s}\right\|_{L^{\infty}(\Omega)} \leq \bar{\kappa}\left\|u_{\varepsilon}\right\|_{L^{1}(\Omega)}\|v\|_{L^{2}(\Omega)}
$$

that is

$$
\left|\int_{\mathbb{R}^{2 n}} \frac{\left(\widetilde{u}_{\varepsilon}(x)-\widetilde{u}_{\varepsilon}(y)\right)(v(x)-v(y))}{|x-y|^{n+2 s}} d x d y\right| \leq \bar{\kappa}\|v\|_{L^{2}(\Omega)}\left\|u_{\varepsilon}\right\|_{L^{1}(\Omega)}
$$

for a suitable $\bar{\kappa}>0$ possibly depending on $k$, but independent of $\varepsilon$. Hence, (3.17) is proved and this ends the proof of Claim 2.

Now, we are ready to show the validity of (3.8), that is

$$
M_{\varepsilon}=\int_{\mathbb{R}^{2 n}} \frac{\left|u_{M}(x)-u_{M}(y)\right|^{2}}{|x-y|^{n+2 s}} d x d y-\lambda_{k, s} \int_{\Omega}\left|u_{M}(x)\right|^{2} d x<S_{s} .
$$

In doing this, we have to take into account that $u_{M}=v+P_{k} \widetilde{v}+t \widetilde{u}_{\varepsilon}$ by Claim 2 and that, in particular, we have to estimate three different contributions coming from $v, P_{k} \widetilde{v}$ and $\widetilde{u}_{\varepsilon}$. With respect to similar calculations carried on in $[13$, Subsection 7.2] and [18, Section 7], here we have to pay attention to the contribution coming from $v$, due to the resonance occurring in this case. Let us show (3.19). 
By Claim 2 we have that

$$
\begin{aligned}
M_{\varepsilon}= & \int_{\mathbb{R}^{2 n}} \frac{\left|u_{M}(x)-u_{M}(y)\right|^{2}}{|x-y|^{n+2 s}} d x d y-\lambda_{k, s} \int_{\Omega}\left|u_{M}(x)\right|^{2} d x \\
= & \int_{\mathbb{R}^{2 n}} \frac{\left|v(x)+P_{k} \widetilde{v}(x)+t \widetilde{u}_{\varepsilon}(x)-v(y)-P_{k} \widetilde{v}(y)-t \widetilde{u}_{\varepsilon}(y)\right|^{2}}{|x-y|^{n+2 s}} d x d y \\
& -\lambda_{k, s} \int_{\Omega}\left|v(x)+P_{k} \widetilde{v}(x)+t \widetilde{u}_{\varepsilon}(x)\right|^{2} d x \\
= & \|v\|_{X_{0}}^{2}+\left\|P_{k} \widetilde{v}\right\|_{X_{0}}^{2}+t^{2}\left\|\widetilde{u}_{\varepsilon}\right\|_{X_{0}}^{2} \\
& +2 t \int_{\mathbb{R}^{2 n}} \frac{\left(\widetilde{u}_{\varepsilon}(x)-\widetilde{u}_{\varepsilon}(y)\right)(v(x)-v(y))}{|x-y|^{n+2 s}} d x d y \\
& -\lambda_{k, s}\left(\|v\|_{L^{2}(\Omega)}^{2}+\left\|P_{k} \widetilde{v}\right\|_{L^{2}(\Omega)}^{2}+t^{2}\left\|\widetilde{u}_{\varepsilon}\right\|_{L^{2}(\Omega)}^{2}\right) \\
& -2 \lambda_{k, s} t \int_{\Omega} \widetilde{u}_{\varepsilon}(x) v(x) d x \\
= & \|v\|_{X_{0}}^{2}+t^{2}\left\|\widetilde{u}_{\varepsilon}\right\|_{X_{0}}^{2}+2 t \int_{\mathbb{R}^{2 n}} \frac{\left(\widetilde{u}_{\varepsilon}(x)-\widetilde{u}_{\varepsilon}(y)\right)(v(x)-v(y))}{|x-y|^{n+2 s}} d x d y \\
& -\lambda_{k, s}\left(\|v\|_{L^{2}(\Omega)}^{2}+t^{2}\left\|\widetilde{u}_{\varepsilon}\right\|_{L^{2}(\Omega)}^{2}\right)-2 \lambda_{k, s} t \int_{\Omega} \widetilde{u}_{\varepsilon}(x) v(x) d x
\end{aligned}
$$

thanks to the orthogonality properties of $v, P_{k} \widetilde{v}$ and $\widetilde{u}_{\varepsilon}$ and also to the definition of $\lambda_{k, s}$. Now, note that by (3.15)

$$
\begin{aligned}
\left\|\widetilde{u}_{\varepsilon}\right\|_{X_{0}}^{2}-\lambda_{k, s}\left\|\widetilde{u}_{\varepsilon}\right\|_{L^{2}(\Omega)}^{2}=\left\|u_{\varepsilon}-P_{k} u_{\varepsilon}\right\|_{X_{0}}^{2}-\lambda_{k, s}\left\|u_{\varepsilon}-P_{k} u_{\varepsilon}\right\|_{L^{2}(\Omega)}^{2} \\
=\left\|u_{\varepsilon}\right\|_{X_{0}}^{2}+\left\|P_{k} u_{\varepsilon}\right\|_{X_{0}}^{2} \\
\quad-2 \int_{\mathbb{R}^{2 n}} \frac{\left(u_{\varepsilon}(x)-u_{\varepsilon}(y)\right)\left(P_{k} u_{\varepsilon}(x)-P_{k} u_{\varepsilon}(y)\right.}{|x-y|^{n+2 s}} d x d y \\
\quad-\lambda_{k, s}\left(\left\|u_{\varepsilon}\right\|_{L^{2}(\Omega)}^{2}+\left\|P_{k} u_{\varepsilon}\right\|_{L^{2}(\Omega)}^{2}\right)+2 \lambda_{k, s} \int_{\Omega} u_{\varepsilon}(x) P_{k} u_{\varepsilon}(x) d x \\
=\left\|u_{\varepsilon}\right\|_{X_{0}}^{2}-\lambda_{k, s}\left\|u_{\varepsilon}\right\|_{L^{2}(\Omega)}^{2} \\
\quad-2 \int_{\mathbb{R}^{2 n}} \frac{\left(u_{\varepsilon}(x)-u_{\varepsilon}(y)\right)\left(P_{k} u_{\varepsilon}(x)-P_{k} u_{\varepsilon}(y)\right)}{|x-y|^{n+2 s}} d x d y \\
\quad+2 \lambda_{k, s} \int_{\Omega} u_{\varepsilon}(x) P_{k} u_{\varepsilon}(x) d x \\
=\left\|u_{\varepsilon}\right\|_{X_{0}}^{2}-\lambda_{k, s}\left\|u_{\varepsilon}\right\|_{L^{2}(\Omega)}^{2}-2\left(\left\|P_{k} u_{\varepsilon}\right\|_{X_{0}}^{2}-\lambda_{k, s}\left\|P_{k} u_{\varepsilon}\right\|_{L^{2}(\Omega)}^{2}\right) \\
=\left\|u_{\varepsilon}\right\|_{X_{0}}^{2}-\lambda_{k, s}\left\|u_{\varepsilon}\right\|_{L^{2}(\Omega)}^{2},
\end{aligned}
$$

thanks to the definition of $P_{k}$. Then, combining (3.16), (3.17), (3.20), Claim 1 and (3.21), we get

$$
\begin{aligned}
M_{\varepsilon}= & \|v\|_{X_{0}}^{2}+t^{2}\left\|\widetilde{u}_{\varepsilon}\right\|_{X_{0}}^{2}+2 t \int_{\mathbb{R}^{2 n}} \frac{\left(\widetilde{u}_{\varepsilon}(x)-\widetilde{u}_{\varepsilon}(y)\right)(v(x)-v(y))}{|x-y|^{n+2 s}} d x d y \\
& -\lambda_{k, s}\left(\|v\|_{L^{2}(\Omega)}^{2}+t^{2}\left\|\widetilde{u}_{\varepsilon}\right\|_{L^{2}(\Omega)}^{2}\right)-2 \lambda_{k, s} t \int_{\Omega} \widetilde{u}_{\varepsilon}(x) v(x) d x
\end{aligned}
$$




$$
\begin{aligned}
= & \|v\|_{X_{0}}^{2}+t^{2}\left\|u_{\varepsilon}\right\|_{X_{0}}^{2}+2 t \int_{\mathbb{R}^{2 n}} \frac{\left(\widetilde{u}_{\varepsilon}(x)-\widetilde{u}_{\varepsilon}(y)\right)(v(x)-v(y))}{|x-y|^{n+2 s}} d x d y \\
& -\lambda_{k, s}\left(\|v\|_{L^{2}(\Omega)}^{2}+t^{2}\left\|u_{\varepsilon}\right\|_{L^{2}(\Omega)}^{2}\right)-2 \lambda_{k, s} t \int_{\Omega} \widetilde{u}_{\varepsilon}(x) v(x) d x \\
\leq & \|v\|_{X_{0}}^{2}-\lambda_{k, s}\|v\|_{L^{2}(\Omega)}^{2} \\
& +t^{2}\left(\left\|u_{\varepsilon}\right\|_{X_{0}}^{2}-\lambda_{k, s}\left\|u_{\varepsilon}\right\|_{L^{2}(\Omega)}^{2}\right)+\widetilde{\kappa}\|v\|_{L^{2}(\Omega)}\left\|u_{\varepsilon}\right\|_{L^{1}(\Omega)},
\end{aligned}
$$

provided $\varepsilon>0$ is sufficiently small and for some $\widetilde{\kappa}>0$, independent of $\varepsilon$. Since $v \in \operatorname{span}\left\{e_{1, s}, \ldots, e_{k-1, s}\right\}$ and (2.10) holds true, by [13, Proposition 2.3] and (3.22), we have

$$
\begin{aligned}
M_{\varepsilon} \leq & \|v\|_{X_{0}}^{2}-\lambda_{k, s}\|v\|_{L^{2}(\Omega)}^{2} \\
& +t^{2}\left(\left\|u_{\varepsilon}\right\|_{X_{0}}^{2}-\lambda_{k, s}\left\|u_{\varepsilon}\right\|_{L^{2}(\Omega)}^{2}\right)+\widetilde{\kappa}\|v\|_{L^{2}(\Omega)}\left\|u_{\varepsilon}\right\|_{L^{1}(\Omega)} \\
\leq & \left(\lambda_{k-1, s}-\lambda_{k, s}\right)\|v\|_{L^{2}(\Omega)}^{2} \\
& +t^{2}\left(\left\|u_{\varepsilon}\right\|_{X_{0}}^{2}-\lambda_{k, s}\left\|u_{\varepsilon}\right\|_{L^{2}(\Omega)}^{2}\right)+\widetilde{\kappa}\|v\|_{L^{2}(\Omega)}\left\|u_{\varepsilon}\right\|_{L^{1}(\Omega)} \\
= & \left(\lambda_{k-1, s}-\lambda_{k, s}\right)\|v\|_{L^{2}(\Omega)}^{2} \\
& +S_{s, \lambda_{k, s}}\left(u_{\varepsilon}\right)\left\|t u_{\varepsilon}\right\|_{L^{2^{*}}(\Omega)}^{2}+\widetilde{\kappa}\|v\|_{L^{2}(\Omega)}\left\|u_{\varepsilon}\right\|_{L^{1}(\Omega)},
\end{aligned}
$$

if $\varepsilon$ is small enough, where $S_{s, \lambda_{k, s}}(\cdot)$ is the function defined as

$$
\begin{aligned}
H^{s}\left(\mathbb{R}^{n}\right) \backslash\{0\} \ni u & \mapsto S_{s, \lambda_{k}, s}(u) \\
& :=\frac{\int_{\mathbb{R}^{2 n}} \frac{|u(x)-u(y)|^{2}}{|x-y|^{n+2 s}} d x d y-\lambda_{k, s} \int_{\mathbb{R}^{n}}|u(x)|^{2} d x}{\left(\int_{\mathbb{R}^{n}}|u(x)|^{2^{*}} d x\right)^{2 / 2^{*}}} .
\end{aligned}
$$

Now, note that by the convexity $\left({ }^{2}\right)$, the monotonicity properties of the integrals, (3.9), Claim 1 and the fact that in $\operatorname{span}\left\{e_{1, s}, \ldots, e_{k, s}\right\}$ all the norms are equivalent, we have

$$
\begin{aligned}
1=\left\|u_{M}\right\|_{L^{2^{*}}(\Omega)}^{2^{*}} & =\int_{\Omega}\left|u_{M}(x)\right|^{2^{*}} d x \\
& \geq \int_{\Omega}\left|t u_{\varepsilon}(x)\right|^{2^{*}} d x+2^{*} \int_{\Omega}\left(t u_{\varepsilon}(x)\right)^{2^{*}-1} \widetilde{v}(x) d x \\
& \geq\left\|t u_{\varepsilon}\right\|_{L^{2^{*}}(\Omega)}^{2^{*}}-2^{*} \bar{c}^{2^{*}-1}\left\|u_{\varepsilon}\right\|_{L^{2^{*}-1}(\Omega)}^{2^{*}-1}\|\widetilde{v}\|_{L^{\infty}(\Omega)} \\
& \geq\left\|t u_{\varepsilon}\right\|_{L^{2^{*}}(\Omega)}^{2^{*}}-\widehat{c}\left\|u_{\varepsilon}\right\|_{L^{2^{*}-1}(\Omega)}^{2^{*}-1}\|\widetilde{v}\|_{L^{2}(\Omega)},
\end{aligned}
$$

for some positive constant $\widehat{c}$, so that

$$
\left\|t u_{\varepsilon}\right\|_{L^{2^{*}}(\Omega)}^{2^{*}} \leq 1+\widehat{c}\left\|u_{\varepsilon}\right\|_{L^{2^{*}-1}(\Omega)}^{2^{*}-1}\|\widetilde{v}\|_{L^{2}(\Omega)}
$$

$\left(^{2}\right)$ If $f$ is a differentiable convex function, then $f(y) \geq f(x)+f^{\prime}(x)(y-x)$. Here we take $f(s)=s^{2^{*}}, x=t u_{\varepsilon}$ and $y=u_{M}=v+t u_{\varepsilon}$. 
for $\varepsilon$ sufficiently small. Moreover, by the Young inequality for any $\sigma>0$ we have

$$
\widetilde{\kappa}\|v\|_{L^{2}(\Omega)}\left\|u_{\varepsilon}\right\|_{L^{1}(\Omega)} \leq \sigma\|v\|_{L^{2}(\Omega)}^{2}+\frac{\widetilde{\kappa}^{2}}{4 \sigma}\left\|u_{\varepsilon}\right\|_{L^{1}(\Omega)}^{2}
$$

for any $\varepsilon>0$. Hence, (3.23), (3.25) and (3.27) give, for $\varepsilon$ small enough

$$
\begin{aligned}
M_{\varepsilon}= & \left(\lambda_{k-1, s}-\lambda_{k, s}\right)\|v\|_{L^{2}(\Omega)}^{2} \\
& +S_{s, \lambda_{k, s}}\left(u_{\varepsilon}\right)\left\|t u_{\varepsilon}\right\|_{L^{2^{*}}(\Omega)}^{2}+\widetilde{\kappa}\|v\|_{L^{2}(\Omega)}\left\|u_{\varepsilon}\right\|_{L^{1}(\Omega)} \\
\leq & \left(\lambda_{k-1, s}-\lambda_{k, s}\right)\|v\|_{L^{2}(\Omega)}^{2} \\
& +S_{s, \lambda_{k, s}}\left(u_{\varepsilon}\right)\left(1+\widehat{c}\left\|u_{\varepsilon}\right\|_{L^{2^{*}-1}(\Omega)}^{2^{*}-1}\|\widetilde{v}\|_{L^{2}(\Omega)}\right) \\
& +\sigma\|v\|_{L^{2}(\Omega)}^{2}+\frac{\widetilde{\kappa}^{2}}{4 \sigma}\left\|u_{\varepsilon}\right\|_{L^{1}(\Omega)}^{2} \\
= & \left(\lambda_{k-1, s}-\lambda_{k, s}+\sigma\right)\|v\|_{L^{2}(\Omega)}^{2} \\
& +S_{s, \lambda_{k, s}}\left(u_{\varepsilon}\right)\left(1+\mathcal{O}\left(\varepsilon^{(n-2 s) / 2}\right)\right)+\mathcal{O}\left(\varepsilon^{n-2 s}\right),
\end{aligned}
$$

thanks to Proposition 2.2. Now, let us choose $\sigma>0$ be such that $\sigma<\lambda_{k, s}-\lambda_{k-1, s}$ (this choice is admissible since $\lambda_{k, s}-\lambda_{k-1, s}>0$ by (2.10)). Then, (3.28) yields

$$
M_{\varepsilon} \leq S_{s, \lambda_{k, s}}\left(u_{\varepsilon}\right)\left(1+\mathcal{O}\left(\varepsilon^{(n-2 s) / 2}\right)\right)+\mathcal{O}\left(\varepsilon^{n-2 s}\right)
$$

as $\varepsilon \rightarrow 0$. Now, let us distinguish the two different cases $n>4 s$ and $n<4 s$.

Case 1. $n>4 s$. By Proposition 2.2 and by definition of $S_{s, \lambda_{k, s}}(\cdot)($ see $(3.24))$ we get

$$
\begin{aligned}
S_{s, \lambda_{k, s}}\left(u_{\varepsilon}\right) & =\frac{\int_{\mathbb{R}^{2 n}} \frac{\left|u_{\varepsilon}(x)-u_{\varepsilon}(y)\right|^{2}}{|x-y|^{n+2 s}} d x d y-\lambda_{k, s} \int_{\Omega}\left|u_{\varepsilon}(x)\right|^{2} d x}{\left(\int_{\Omega}\left|u_{\varepsilon}(x)\right|^{2^{*}} d x\right)^{2 / 2^{*}}} \\
& \leq \frac{S_{s}^{n /(2 s)}+\mathcal{O}\left(\varepsilon^{n-2 s}\right)-\lambda_{k, s} C_{s} \varepsilon^{2 s}}{\left(S_{s}^{n /(2 s)}+\mathcal{O}\left(\varepsilon^{n}\right)\right)^{2 / 2^{*}}} \leq S_{s}+\mathcal{O}\left(\varepsilon^{n-2 s}\right)-\lambda_{k, s} C_{s} \varepsilon^{2},
\end{aligned}
$$

as $\varepsilon \rightarrow 0$. Thus, as a consequence of this and of (3.29) we deduce

$$
\begin{aligned}
M_{\varepsilon} & \leq\left(S_{s}+\mathcal{O}\left(\varepsilon^{n-2 s}\right)-\lambda_{k, s} C_{s} \varepsilon^{2}\right)\left(1+\mathcal{O}\left(\varepsilon^{(n-2 s) / 2}\right)\right)+\mathcal{O}\left(\varepsilon^{n-2 s}\right) \\
& =S_{s}+\mathcal{O}\left(\varepsilon^{n-2 s}\right)-\lambda_{k, s} C_{s} \varepsilon^{2}=S_{s}+\varepsilon^{2 s}\left(\mathcal{O}\left(\varepsilon^{n-4 s}\right)-\lambda_{k, s} C_{s}\right)<S_{s},
\end{aligned}
$$

provided $\varepsilon>0$ is sufficiently small. Hence, (3.19) holds true. This concludes the proof of Proposition 3.1 in the case when $n>4 s$.

Case 2. $n<4 s$. Again by Proposition 2.2 and by definition of $S_{s, \lambda_{k, s}}(\cdot)$ we have

$$
\begin{aligned}
S_{s, \lambda_{k, s}}\left(u_{\varepsilon}\right) & \leq \frac{S_{s}^{n /(2 s)}+\mathcal{O}\left(\varepsilon^{n-2 s}\right)-\lambda_{k, s} C_{s} \varepsilon^{n-2 s}+\mathcal{O}\left(\varepsilon^{2 s}\right)}{\left(S_{s}^{n /(2 s)}+\mathcal{O}\left(\varepsilon^{n}\right)\right)^{2 / 2^{*}}} \\
& \leq S_{s}+\mathcal{O}\left(\varepsilon^{n-2 s}\right)-\lambda_{k, s} \widetilde{C}_{s} \varepsilon^{n-2 s}+\mathcal{O}\left(\varepsilon^{2 s}\right),
\end{aligned}
$$


as $\varepsilon \rightarrow 0$. Thus, by this and (3.29) we deduce

$$
\begin{aligned}
M_{\varepsilon} & \leq\left(S_{s}+\mathcal{O}\left(\varepsilon^{n-2 s}\right)-\lambda_{k, s} \widetilde{C}_{s} \varepsilon^{n-2 s}+\mathcal{O}\left(\varepsilon^{2 s}\right)\right)\left(1+\mathcal{O}\left(\varepsilon^{(n-2 s) / 2}\right)\right)+\mathcal{O}\left(\varepsilon^{n-2 s}\right) \\
& =S_{s}+\mathcal{O}\left(\varepsilon^{n-2 s}\right)-\lambda_{k, s} \widetilde{C}_{s} \varepsilon^{n-2 s}+\mathcal{O}\left(\varepsilon^{2 s}\right) \\
& =S_{s}+\varepsilon^{n-2 s}\left(\mathcal{O}(1)-\lambda_{k, s} \widetilde{C}_{s}\right)+\mathcal{O}\left(\varepsilon^{2 s}\right)<S_{s},
\end{aligned}
$$

if $\lambda_{k, s}$ is large enough, say $\lambda_{k, s}>\lambda_{s}>0$ and provided $\varepsilon>0$ is sufficiently small. Thus, (3.19) is verified. Ultimately, the proof of Proposition 3.1 is concluded.

Finally, we can prove Theorem 1.1 as an application of classical minimax theorems.

Proof of Theorem 1.1. Now, Theorem 1.1 easily follows from the Linking Theorem (see [11], [12]), thanks to Propositions 2.3, 2.4 and 3.1.

Acknowledgements. The author would like to thank Professor Marco Degiovanni for interesting and useful discussions.

\section{REFERENCES}

[1] A. Ambrosetti And P. Rabinowitz, Dual variational methods in critical point theory andapplications, J. Funct. Anal. 14 (1973), 349-381.

[2] G. Arioli, F. Gazzola, H.-Ch. Grunau and E. Sassone, The second bifurcation branch for radial solutions of the Brezis-Nirenberg problem in dimension four, NoDEA Nonlinear Differential Equations Appl. 15 (2008), 69-90.

[3] B. Barrios, E. Colorado, A. De Pablo and U. Sanchez, On some critical problems for thefractional Laplacian operator, J. Differential Equations 252 (2012), 6133-6162.

[4] H. Brezis and L. Nirenberg, Positive solutionsof nonlinear elliptic equations involving critical Sobolev exponents, Comm. Pure Appl. Math. 36 (1983), 437-477.

[5] A. Capozzi, D. Fortunato and G. Palmieri, An existence result for nonlinear elliptic problems involving critical Sobolev exponent, Ann. Inst. H. Poincaré Anal. Non Linéaire 2 (1985).

[6] A. Cotsiolis And N. Tavoularis, Best constants for Sobolev inequalities for higher order fractional derivatives, J. Math. Anal. Appl. 295 (2004), 225-236.

[7] E. Di Nezza, G. Palatucci and E. Valdinoci, Hitchhiker's guide to the fractional Sobolev spaces, Bull. Sci. Math. 136 (2012), 521-573.

[8] F. Gazzola And H.-CH. Grunau, On the role of space dimension $n=2+2 \sqrt{2}$ in the semilinear Brezis-Nirenberg eigenvalue problem, Analysis (Munich) 20 (2000), 395-399.

[9] F. Gazzola AND B. RuF, Lower-order perturbations of critical growth nonlinearities in semilinear elliptic equations, Adv. Differential Equations 2 (1997), 555-572.

[10] O. KaVIAN, Introduction à la théorie des points critiques et applications aux problèmes elliptiques, Mathématiques \& Applications, Springer-Verlag, Paris, 1993.

[11] P.H. Rabinowitz, Some critical point theorems and applications to semilinear elliptic partial differential equations, Ann. Scuola Norm. Sup. Pisa Cl. Sci. (4) 5 (1978), 215-223.

[12] _ Minimax methods in critical point theory with applications to differential equations, CBMS Reg. Conf. Ser. Math., vol. 65, American Mathematical Society Providence, RI, 1986. 
[13] R. Servadei, The Yamabe equation in a non-local setting, Adv. Nonlinear Anal. 2 (2013), 235-270.

[14] R. Servadei AND E. VAldinoci, Lewy-Stampacchia type estimates for variational inequalities driven by (non)local operators, Rev. Mat. Iberoam. 29 (2013), 1091-1126.

[15] _ Mountain Pass solutionsfor non-local elliptic operators, J. Math. Anal. Appl. 389 (2012), 887-898.

[16] Variational methods for non-local operators of elliptic type, Discrete Contin. Dyn. Syst. 33 (2013), 2105-2137.

[17] R. Servadei And E. VAldinoci, The Brezis-Nirenberg result for the fractional Laplacian, Trans. Amer. Math. Soc. (to appear).

[18] A Brezis-Nirenberg result for non-local critical equations in low dimension, Commun. Pure Appl. Anal. 12 (2013), 2445-2464.

[19] , Fractional Laplacian equations with critical Sobolev exponent, preprint, http: //www.ma.utexas.edu/mp_arc-bin/mpa?yn=12-58.

[20] M. Struwe, Variational Methods, Applications to Nonlinear Partial Differential Equations and Hamiltonian Systems, Ergebnisse der Mathematik und ihrer Grenzgebiete, vol. 3, Springer Verlag, Berlin-Heidelberg, 1990.

[21] J. TAN, The Brezis-Nirenberg type probleminvolving the squareroot of the Laplacian, Calc. Var. Partial Differential Equations 36 (2011), 21-41.

[22] M. Willem, Minimax Theorems, Progress in Nonlinear Differential Equationsand their Applications, vol. 24, Birkhäuser, Boston, 1996.

[23] D. Zhang, On multiple solutions of $\Delta u+\lambda u+|u|^{4 /(n-2)} u=0$, Nonlinear Anal. 13 (1989), 353-372.

Raffaella Servadei

Dipartimento di Matematica e Informatica

Università della Calabria

Ponte Pietro Bucci 31 B

87036 Arcavacata di Rende (Cosenza), ITALY

E-mail address: servadei@mat.unical.it 